\title{
Ultrahigh impedance method to assess electrostatic accelerator performance
}

\author{
Nikolai R. Lobanov, Peter Linardakis, and Dimitrios Tsifakis \\ The Department of Nuclear Physics, Research School of Physics and Engineering, \\ The Australian National University, Canberra, Australia
}

(Received 26 February 2015; published 30 June 2015)

\begin{abstract}
This paper describes an investigation of problem-solving procedures to troubleshoot electrostatic accelerators. A novel technique to diagnose issues with high-voltage components is described. The main application of this technique is noninvasive testing of electrostatic accelerator high-voltage grading systems, measuring insulation resistance, or determining the volume and surface resistivity of insulation materials used in column posts and acceleration tubes. In addition, this technique allows verification of the continuity of the resistive divider assembly as a complete circuit, revealing if an electrical path exists between equipotential rings, resistors, tube electrodes, and column post-to-tube conductors. It is capable of identifying and locating a "microbreak" in a resistor and the experimental validation of the transfer function of the high impedance energy control element. A simple and practical fault-finding procedure has been developed based on fundamental principles. The experimental distributions of relative resistance deviations $(\Delta R / R)$ for both accelerating tubes and posts were collected during five scheduled accelerator maintenance tank openings during 2013 and 2014. Components with measured $\Delta R / R> \pm 2.5 \%$ were considered faulty and put through a detailed examination, with faults categorized. In total, thirty four unique fault categories were identified and most would not be identifiable without the new technique described. The most common failure mode was permanent and irreversible insulator current leakage that developed after being exposed to the ambient environment. As a result of efficient in situ troubleshooting and fault-elimination techniques, the maximum values of $|\Delta R / R|$ are kept below $2.5 \%$ at the conclusion of maintenance procedures. The acceptance margin could be narrowed even further by a factor of 2.5 by increasing the test voltage from $40 \mathrm{~V}$ up to $100 \mathrm{~V}$. Based on experience over the last two years, resistor and insulator failures are less common now due to the routine use of the noninvasive ultrahigh impedance technique.
\end{abstract}

DOI: 10.1103/PhysRevSTAB.18.060101

PACS numbers: 29.20.Ba

\section{INTRODUCTION}

In electrostatic accelerators, the high-voltage potential is generated by mechanical transfer of charge from ground to the high-voltage terminal. Ions travel through evacuated acceleration tubes constructed of dense, low outgassing, insulating rings sealed to metal electrodes. Glass insulating rings sealed with polyvinylacetate, together with aluminium, titanium, or stainless steel electrodes are widely used [1]. Tubes developed by the National Electrostatic Corporation (NEC) use high-density alumina ceramic insulating rings. A voltage gradient between electrodes is established by resistors conducting current from the highvoltage terminal. It is generally accepted that a reliable gradient of about $1 \frac{2}{3} \mathrm{MV} / \mathrm{m}$ can be achieved with a modern acceleration tube [2].

Below a terminal voltage of $5 \mathrm{MV}$, acrylic plastic plates are used as the insulating mechanical support column post around the acceleration tubes. Above $5 \mathrm{MV}$, alumina

Published by the American Physical Society under the terms of the Creative Commons Attribution 3.0 License. Further distribution of this work must maintain attribution to the author $(s)$ and the published article's title, journal citation, and DOI. ceramic insulators bonded with aluminium to titanium electrodes are used to form posts about $0.5 \mathrm{~m}$ long. Stainless steel hoops are positioned along the post and are connected to the acceleration tube resistor divider.

For example, the configuration of the vertically orientated 14UD accelerator produced by National Electrostatic Corporation is based on 28 19-inch-long live modules (units). Units 1-14 are grouped into the low-energy end, units 15-28 into the high-energy end with the high-voltage terminal in between.

The electrical structure of each unit consists of four ceramic posts and three and half acceleration tubes, using high-voltage resistors and the voltage grading system. Each unit is designed to withstand an operational voltage of up to $1 \mathrm{MV}$ at $\sim 100$ psi pressure of sulphur hexafluoride $\left(\mathrm{SF}_{6}\right)$ gas. In each unit, there are 74 acceleration tube resistors with a total resistance of $R_{\Sigma}=22.2 \mathrm{G} \Omega$ across 37 ceramic gaps and 36 post resistors with $R_{\Sigma}=32.9 \mathrm{G} \Omega$ across 18 gaps in each of four posts.

The high-voltage performance of an electrostatic accelerator depends on good control of the electric field distribution across its structure, which is dependent on the voltage distribution system. In the 1980s, corona point grading provided an adequate solution. One disadvantage 
of the corona point system is that the corona extinguishes when the gap voltage reduces below the threshold, limiting accelerator operation at low voltage. More drastically, the corona produces corrosive $\mathrm{SF}_{6}$ breakdown products that attack the Pelletron charging chains and ceramic insulators. Thus, modern voltage grading systems are instead based on resistors.

However, these too can have issues. Spark events are triggered when a region of high electrical stress in the accelerator breaks down. This can take place either in the vacuum inside the acceleration tube or in the insulation gas environment in the external region of the acceleration tube or post. The large amount of electrostatic energy stored in the accelerator is transmitted during a spark and can cause resistors to fail. The development of robust resistor grading systems requires the implementation of protection strategies to suppress the coupling of transient energy to the resistors. The important components for such protection are spark gaps, minimization of aerial coupling, shielding, structural insulation, and the property of the resistors themselves [3].

In the 1960s, a carbon resistor values changed by more than $25 \%$ after a short exposure to electric stress and sparks. In accelerators with inclined-field accelerating tubes, this caused beam transmission problems because the beam deflected from one side of the axis to the other. In addition, increases in resistor values overstressed the insulating gap and lowered the maximum voltage of the accelerator. Decreases in resistance exposed the remaining resistors to a higher than nominal voltage, triggering sparks. Resistor assemblies failed frequently and the cost of constant replacement became too high. This drove a need to improve reliability and to reduce costs.

Resistors constructed of metal-oxide-coated ceramic cylinders displaced the carbon resistors. Uncoated solid ceramic-core resistors are now the preferred option for use in electrostatic accelerators and are available commercially. The metal-oxide resistive layer is often manufactured in a spiral pattern or low-inductance patterned resistors are available in order to reduce coupling to transients. However, even resistors with a spiral pattern exhibited robust performance in accelerators.

The ideal resistor system depends on various trade-offs such as the cost, maintenance, and the value of accelerator utilization. High reliability is the main consideration in large accelerators, where beam quality is crucial and system down time is intolerable. Nearly all large tandem electrostatic accelerators use independent voltage grading of the posts and the tube in order to minimize tube damage. There are a total of 3058 resistors, 2016 ceramic gaps in posts, and 1036 ceramic gaps in acceleration tubes in a large tandem accelerator like the Australian National University's (ANU's) 14UD.

This paper has been organized into five main sections. Section II outlines resistor failure modes and Sec. III continues on to describe the general concept of high impedance measurement to characterize resistor performance. Section IV introduces the experimental design of an optimal test for in situ resistor characterization, together with the protocols for collecting data and the data analysis procedures. Section V presents key experimental results on a high-voltage grading system, which have been systematically collected during four scheduled maintenance openings of the ANU's 14UD accelerator over the last few years. Finally, the Sec. VI presents interpretation of test results in view of what is discussed throughout the paper.

\section{RESISTOR FAILURE MODES}

Even though high-voltage resistors are comparatively reliable, faults can and do occur. Therefore resistors must be tested and maintained to ensure reliable and predictable accelerator performance. Typical failure modes can be classified by severity as follows [4]:

A. An explosion can happen when excessive stored energy is absorbed into the resistor during an accelerator spark event. This generally results when the geometry of resistor assembly provides coupling to excessive transient fields.

B. Mechanical failure can result from excessive or prolonged strain or exposure to vibration or temperature gradient. Caddock and Welwyn branded resistors are subject to this because of their small diameter $(10 \mathrm{~mm}$ and $6 \mathrm{~mm}$, respectively).

C. Exposure to a corona discharge can cause erosion of the conductive layer and change the resistance.

D. An open circuit can be caused by failure of the external conductors and connectors or as a result of a mechanical break.

E. The most troublesome phenomenon is a "microbreak" detectable only by low-voltage measurement. The microbreak might be caused by localized vaporization of the resistor film due to high-instantaneous voltage. Vaporization would leave rounded edges in the resistive film as shown in Fig. 1. A Welwyn resistor with a defect in conductive layer is shown in Fig. 1. Breaks are most likely to occur near the end caps where the self-inductance is highest. Also, corona discharge from the sharp edge of the end caps may burn through the film. An alternative mechanism for a microbreak is magnetostrictive shock, which could leave sharp edges in the film as a result of the chipping in the conductive layer.

F. Changes in film resistance occur in nearly all film resistors. Usually, resistors all "age" with resistance values decreasing by a few percent during the first weeks of operation in an accelerator under stressed conditions. The aging is attributed by Caddock to irreversible "welding" together of conductive islands in the film material. Resistors are unlikely to age consistently, so the uniformity of the accelerator voltage gradient is affected. 


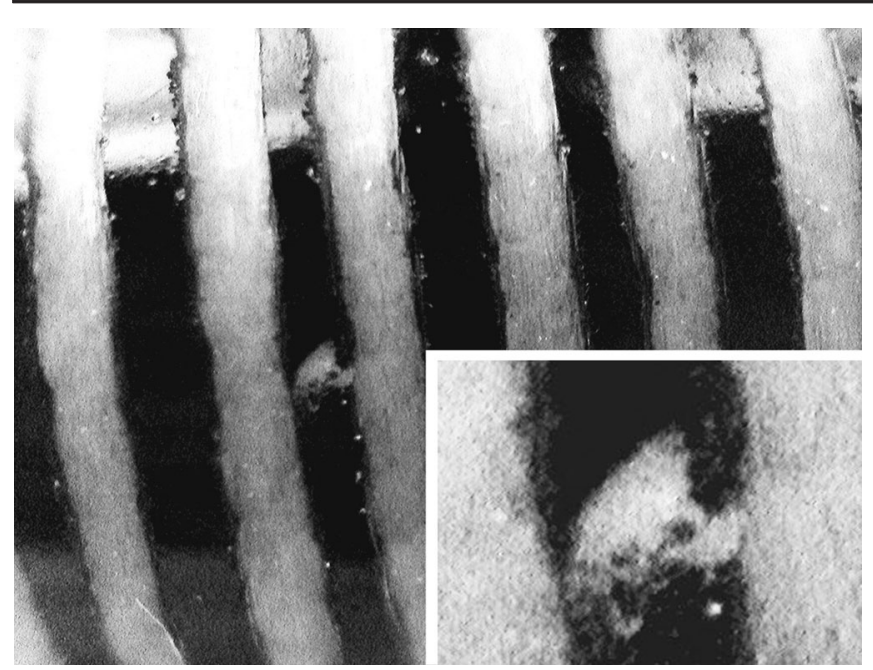

FIG. 1. A discontinuity or "microbreak" in the resistor film (black) is seen in the third turn from the left on a Welwyn $982 \mathrm{M} \Omega$ post resistor. Rounded edges of the resistor film in the microbreak suggest possible localized vaporization of the film due to high-instantaneous voltage. Inset shows the magnified image of the microbreak.

G. A short circuit could result from mechanical failure or from conductive debris bridging across the spark gap. Alternatively, a permanent reduction of resistance may be caused by long exposure to corona discharges or to accelerator spark events.

Insulators in the voltage grading system must be also tested and maintained to ensure reliable operation. Typical failure modes of insulation components are the following:

$\mathrm{H}$. Mechanical failure varying from surface flaking to crisscrossed cracking to complete separation of the bond between the ceramic and titanium. Although electric breakdown causes the ultimate failure of the electrical insulation, electrical stress is not the dominating aging factor. It is believed that the aging mechanism is dominated by mechanical stress caused by vibration, spark events, and by the different thermal expansion coefficients of the materials involved. Since insulators often form part of the mechanical supporting structure of an electrostatic accelerator, failure can be a dangerous and expensive.

I. Permanent leakage current caused by dielectric failure. Electrical tracking can occur as a conductive path along a surface or bulk is created over time due to environmental conditions such as temperature, humidity, radiation, and pressure.

J. Insulator leakage current developed after being exposed to the ambient, atmospheric environment. Typically it is associated with hairline cracking and manifests as surface current leaks or due to humidity or surface contamination.

Open circuit condition can be caused by failure of external conductors and connectors as follows:

$\mathrm{K}$. Breaks in the electrical path between equipotential rings and post electrodes.
L. Lack of continuity or poor electrical connection between post electrodes and post-to-tube conductors.

M. Lack of continuity or poor electrical connection between post-to-tube conductors and tube electrodes.

N. A break in the electrical path between a pair of resistors.

Finally, the unbalanced voltage distribution may occur due to fault conditions during installation of the resistors. For example, it could be caused by selection of resistors with an incorrect value.

O. Incorrect resistor values.

A survey of experiences with resistor assemblies from different laboratories is given in [3]. The survey spans different types of performance evaluations, various operation voltages spanning from few megavolts up to $25 \mathrm{MV}$, and a wide variation of spark event frequency. Ignoring the differences, as a first approximation, good performance is indicated by a relative resistance change of $\Delta R / R<5 \%$.

Resistor assemblies act as aerials that will absorb radiated rf power during a spark event. The laboratories mentioned in [3] favor short aerials and open packing and therefore convenient access to the resistors. The reported data shows a weak correlation between the shielding technique and performance. However, coaxial shields and thimble electrodes are preferred where high electrical stress is encountered.

\section{HIGH-VOLTAGE TESTING OF RESISTORS}

Considerable information about the condition of resistors condition can be gained by tracking values over time with a high-voltage megohmmeter.

Reference [4] describes a setup based on a $3 \mathrm{kV}$ device equipped with a high-impedance probe for safe operation. The three-digit $\mathrm{M} \Omega$ reading is produced in an analogue chip from the actual applied voltage and current with an accuracy of $\pm 1 \mathrm{M} \Omega$, which corresponds to an accuracy reading of a typical resistor value of $300 \mathrm{M} \Omega$ of $\Delta R / R= \pm 0.33 \%$.

At the ANU, a Danbridge JP30A $30 \mathrm{kV}$ nondestructive insulation tester is used for high-voltage evaluation of resistors. The tester provides a de voltage adjustable up to $30 \mathrm{kV}$. The output voltage and current are displayed on separate meters with an accuracy of $\pm 5 \%$. Any ionization due to corona is audible through a built-in loudspeaker. The rear panel connector provides both voltage and current meter analogue outputs ( $+5 \mathrm{~V}$ for full scale meter reading). An accuracy of $\pm 1 \%$ is possible by using these analogue outputs. The 14UD accelerator column post unit structure is arranged in double units, with each double unit consisting of six tubes having 11 electrodes and one tube with 8 electrodes. The spacing between electrodes on the column post and the tube is $25.4 \mathrm{~mm}$ and $12.7 \mathrm{~mm}$, respectively. The entire accelerator has seven double units in the lowenergy section and six double units and two single units in 


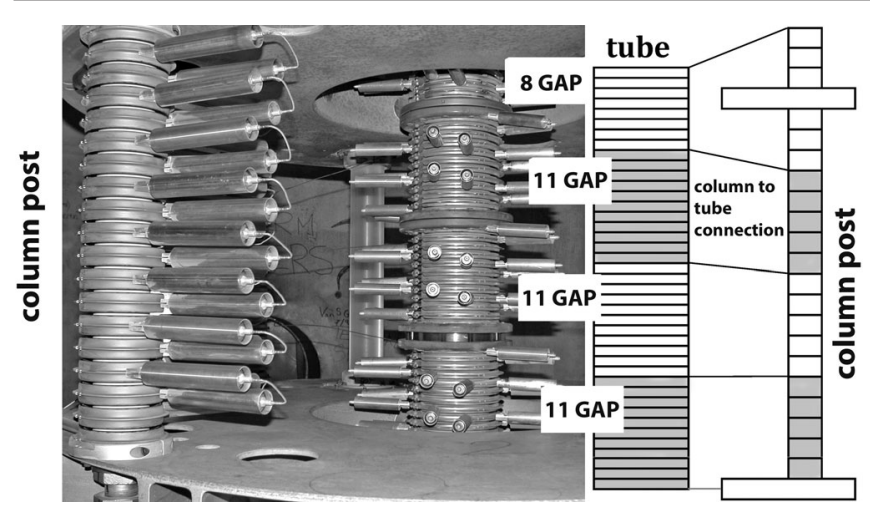

FIG. 2. Photograph (left) and schematic sketch (right) of half of the 14UD double unit. The flexible connections between pairs of tube resistors are removed. The unit is completely stripped of equipotential rings.

the high-energy section. The schematic configuration of a double unit is shown in Fig. 2.

Each end of 11 gap tubes is connected to the post electrodes by means of $1 / 16$-inch spring copper wire. The ANU design employs a pair of $40 \mathrm{kV}, 112 \mathrm{~mm}$ long Welwyn resistors for each post gap. The resistance value is $982 \mathrm{M} \Omega$ in 5 gap sections of the column post and $575 \mathrm{M} \Omega$ in 6 gap sections. Tubes employ pairs of $20 \mathrm{kV}, 61-\mathrm{mm}-$ long $300 \mathrm{M} \Omega$ resistors throughout the entire accelerator. At an operational voltage of $1 \mathrm{MV}$ per unit at $\sim 100 \mathrm{psi}$ pressure of $\mathrm{SF}_{6}$, the maximum voltage between adjacent equipotential rings would be $66.7 \mathrm{kV}$, corresponding to a voltage across a single column post resistor of $33.4 \mathrm{kV}$. The maximum voltage drop per tube ceramic gap would be $27 \mathrm{kV}$ and therefore the voltage across a single tube resistor is $13.5 \mathrm{kV}$.

During testing of groups of resistors, $30 \mathrm{kV}$ is applied to an assembly incorporating an 11 gap tube in parallel with five column post gaps or an 8 gap tube in parallel with 6 gaps on the post. In an ambient air environment, the maximum test voltage is limited to below $7 \mathrm{kV}$. This is lower than the nominal voltage because all spark gaps are designed to operate in $\mathrm{SF}_{6}$ gas at 100 psi. The reduced test voltage will not always pinpoint bad resistors, especially if they may break down under operational voltage. The resistance of an entire assembly is determined from an applied test voltage $U_{T}=30 \mathrm{kV}$ and measured current $I_{R}$. Consider a chain of $N$ identical resistors of value $R$ in series with an applied voltage $U_{T}$. The measured current is given by the equation $I_{R}=U_{T} / N R$. If the value of only one resistor is changed by $\Delta R$, the measured current becomes $I_{\Delta R}=U_{T} /(\Delta R+N R)$. Therefore, the current varies by $\Delta I=I_{R}-I_{\Delta R}$ and the relative resistance change is then

$$
\Delta R / R=N^{2} R \Delta I /\left(U_{T}-N R \Delta I\right) .
$$

The resolution of this method is limited by the accuracy of current measurement $\Delta I / I=1 \%$ or $\Delta I=10^{-7} \mathrm{~A}$ at a

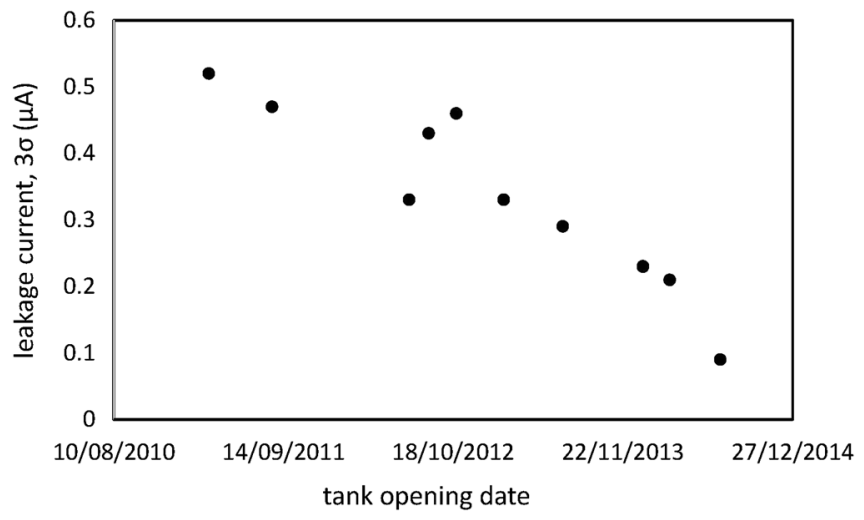

FIG. 3. Standard exit high voltage test result variation conducted on eleven gap tubes over the last few years on the ANU's 14UD electrostatic accelerator.

full scale of $10 \mu \mathrm{A}$. For an 11 gap tube structure with $N=11$ and $R=2 \times 300=600 \mathrm{M} \Omega$, the calculated $\Delta R / R_{11 G T}=24.7 \%$. For a corresponding 5 gap post structure with $N=5$ and $R=2 \times 982=1964 \mathrm{M} \Omega$, the calculated $\Delta R / R_{5 G P}=16.9 \%$. The resolution is considerably higher than the commonly accepted criteria $\Delta R / R<5 \%$.

Nevertheless, over the last few years the margin of acceptance in our laboratory has narrowed a great deal. Figure 3 shows the standard deviation of $30 \mathrm{kV}$ highvoltage exit test results conducted on 11 gap tubes.

During the measurement of high-impedance resistor/ insulator assemblies, time-dependant currents occur when a $\mathrm{dc}$ voltage is applied. The total current read on the meter of the test set is a composite of three currents: the leakage current through the resistor $I_{R}$, the capacitive current $I_{C}$, and the absorption current $I_{a}$. The resistor current $I_{R}$ reaches a constant value in a period of time. The capacitive current $I_{C}$ is caused by the charging of the capacitance of the assembly. This current decreases to zero in a few seconds. The absorption current $I_{a}$ is caused by changes in the molecular structure of the insulation material (ceramic or glass). In some cases it falls to zero in a few seconds, but in other cases it can persist for hours, which might indicate a fault with the insulators. When the capacitive and absorption currents have fallen to almost zero, the total current and resistor current are the same and accurate measurement becomes possible. Time-dependent currents cause problems when one is testing assemblies with high capacitance. Fortunately, the capacitance of resistor/ insulator assemblies is not significant-of the order of few tens of picofarads-and the measurement can be done in a few seconds.

In a true high voltage test, the nominal high voltage is applied between a resistor and its metallic shielding as shown in Fig. 4. This is an invasive test since the equipotential rings must be removed in order to gain access to the resistors. In addition, one should remove 


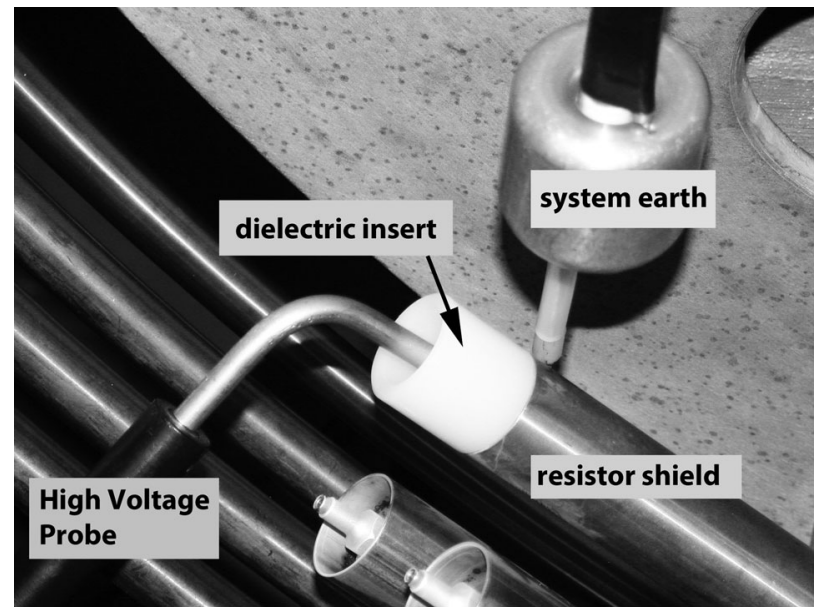

FIG. 4. In-machine high-voltage individual resistor testing procedure. The dielectric insert is made of nylon 6 . The conductive leads between the neighbor resistors are removed.

the conductive leads between the neighboring resistors and use a special dielectric insert to suppress breakdown across the resistor spark gap. The resulting current that flows through the resistor is monitored by the tester. In addition to measuring the resistance, the test can also be performed to detect material and workmanship defects [5].

When a resistor assembly is operated under ambient conditions, environmental factors such as humidity, dirt, vibration, and contaminants can affect the measurements. Moreover, the hydroscopic deposits from $\mathrm{SF}_{6}$ breakdown products and dust from mechanical systems intensify this problem. Any corona discharge can compromise the accuracy of measurements at high voltage in ambient air. Corona is usually observed where a discharge occurs due to the breakdown of a small area of the insulation. The extra current drawn from the supply is recorded by the output meter and consequently gives a false lower reading of resistance. Furthermore, the current is unstable, which tends to cause the meter to read erratically. Therefore, in-machine testing using the high-voltage diagnostic is limited to discovering gross combined failures such as mechanical and electrical. The condition of individual components is not identified. In addition, high voltage tests quite often fail to diagnose a microbreak. With kilovolts applied, the current is carried across the microbreak by an arc and initially there may be no apparent change in resistance. Eventually the microbreak erodes completely and a permanent open-circuit develops.

A low-voltage measurement of very high-value resistors is reported in [4]. A Fluke model 8020 digital multimeter is used utilizing a nanosiemens conductivity range. A conductance function allows fast, noise-free resistance measurements up to $10 \mathrm{G} \Omega$. Ordinary resistance measurements within this range are plagued by noise pickup, require careful shielding, and are limited by low input impedance of the device itself, which is $10 \mathrm{M} \Omega$. By measuring the resistance in terms of conductance, high-value resistors are to be disconnected from assembly; therefore, this test requires dismounting the equipotential rings to get access inside a 14UD unit. Although the microbreak is detectable by low-voltage measurement, this technique is not able to provide information about its location on the resistive film.

The development of a technique for the direct experimental validation of the transfer function (TF) of the energy control element of electrostatic accelerators is important, since the mathematical analysis of electrostatic accelerators is problematic. The TF is used to design the energy feedback control system delivering high-resolution particle beams with the energy stability of the order $10^{-5}$. Since the accelerator impedance is of the order of 100 gigaohms, a direct measurement of the TF is a big challenge. Therefore, it would be advantageous if the de technique developed for resistor diagnostics could be applied to ac time domain measurements [6].

Table I summarizes resistor and insulation components failure modes and the diagnostic techniques employed in different laboratories. The optimal test should allow the full

TABLE I. The summary of resistor and insulation components failure modes and diagnostic techniques used in accelerator laboratories. An optimal test should allow the full set of failure modes to be identified with high accuracy and with minimum time.

\begin{tabular}{|c|c|c|c|c|c|}
\hline Test criteria & High-voltage group test & High-voltage single test & Low-voltage conductivity test & Failure mode & Ref. \\
\hline Change of resistance & $\checkmark$ & $\checkmark$ & $\checkmark$ & $\mathrm{C}, \mathrm{F}, \mathrm{O}$ & [5] \\
\hline Open circuit & $\checkmark$ & $\checkmark$ & $\checkmark$ & $\mathrm{A}, \mathrm{B}, \mathrm{D}$ & \\
\hline "Microbreak" & & & $\checkmark$ & $\mathrm{D}$ & [4] \\
\hline "Microbreak" spotting & & & & $\mathrm{D}$ & \\
\hline Short circuit & $\checkmark$ & $\checkmark$ & $\checkmark$ & G & \\
\hline Insulator failure & & & & $\mathrm{H}$ & \\
\hline Insulator leakage & $\checkmark$ & $\checkmark$ & & I, J & \\
\hline Continuity test & & & $\checkmark$ & $\mathrm{K}, \mathrm{L}, \mathrm{M}, \mathrm{N}$ & \\
\hline Noninvasive & $\checkmark$ & & & & \\
\hline Immune to ambient & & & $\checkmark$ & & \\
\hline Fast & $\checkmark$ & & & & \\
\hline Transfer function & & & & & [6] \\
\hline
\end{tabular}


set of failure modes to be identified with high accuracy and with minimum time. All other tests listed in the table require a greater number of experimental runs to estimate the parameters with the same precision as an optimal test in a single run. In practical terms, an optimal test can reduce the costs and time of experimentation. The development of a novel diagnostic technique for an electrostatic accelerator high-voltage grading system fulfilling the optimum test criteria is the main purpose of the investigation of this paper.

\section{ULTRAHIGH IMPEDANCE VOLTMETER FOR ELECTROSTATIC ACCELERATOR APPLICATIONS}

A good voltage measuring technique that can be applied to electrostatic accelerators should be able to measure voltage without changing it. Many such systems have been developed over the years. Generally, measurements are either noncontact or contact. The Kelvin probe, the rotating vane field meter, and the induction probe are well-known examples of noncontacting electrostatic voltage measurement instruments. Lack of physical contact between the object under investigation (OUI) and the sensor of the instrument makes certain that no charge is transferred through the air gap acting as an ultrahigh-impedance media.

The Kelvin probe is a noncontact, nondestructive vibrating capacitor device used to measure the work function of conducting materials or surface potential of semiconductor or insulating surfaces. The most important factors that determine the accuracy of Kelvin probes are (i) sensitivity to electromagnetic and mechanical noise, and (ii) response to stray capacitive coupling to the OUI and to distributed capacitances of the surrounding environment and spacing problems such as edge effects and nonuniform field distribution. Therefore the use of Kelvin probes is complicated and infeasible for large-scale accelerator structures.

In a rotating vane field meter, the field sensing plate is placed behind a grounded rotating blade. The blade periodically shields the sensing plate from the electric field. As a result, the induced charge on the sensing plate is modulated. Usually, the field meter is calibrated in a particular field distribution, such as a uniform field. Unless the field between the meter and charged OUI is similar to the calibration field, the voltage reading (or field reading) is probably not the voltage of the charged object. This is certainly true for accelerator high-voltage grading structures characterized by nonuniform fields.

The fundamental principle of operation for measuring an electric field using an induction probe is to allow a conductive plate or antenna to equilibrate with the local field, then measure the voltage on the plate. An electrometer is used to measure the voltage or charge on the induction plate. Unfortunately, induction probes are not recommended for electric field measurements, since they require frequent rezeroing in shielded conditions and consequently cannot be used for measurements over extended periods of time. Also, they are highly susceptible to ambient space charges. Usually, all noncontact devices measure the average voltage over a "spot size" of approximately $0.5-2 \mathrm{~cm}^{2}$. In many applications, such as electrostatic accelerators, a smaller spot size measurement would be extremely useful.

This can be accomplished by using a new class of voltmeters. A conventional voltmeter utilizes a buffer amplifier, an analogue-to-digital converter, and a display. The voltmeter employs two contacting passive probes and has an input impedance of up to $100 \mathrm{M} \Omega$. In high-impedance applications, the input resistance and input capacitance of a conventional voltmeter rapidly drains the charge from the OUI. As an example, there can be a need to measure the voltage distribution on a chain of grading resistors of the accelerator. Commonly, charge levels of a few nanocolombs can be stored on a distributed grading system capacitance of $C \sim 10 \mathrm{pF}$. Assume that the resistive chain consisting of $R=1 \mathrm{G} \Omega$ is supplied a constant current of $I=0.1 \mu \mathrm{A}$ and then a common multimeter is connected across one resistor to measure voltage across it. The measured voltage initially indicates a voltage of $R \times I=100 \mathrm{~V}$ but soon the meter voltage begins to drop in value. Although the shunt impedance of the system is partially accountable for this decay in voltage, the finite resistance of the multimeter is mostly responsible. If the multimeter has a resistance of $r=100 \mathrm{M} \Omega$, the classical exponential drop in the voltage is given by $V_{(t)}=12 \mathrm{e}^{-t / \tau}$ where $\tau=r R C /(r+R) \approx 10^{-4}$ sec is the time constant of the response, which is very short. The initial voltage, $V_{i}=100 \mathrm{~V}$, will drop very quickly to a measured value, $V_{m}=100 r /(r+R) \approx 9.1 \mathrm{~V}$. This illustrates why ordinary multimeters do not have the necessary high-megaohm input resistances for measurement of this type. Electrometers, though, have input resistances up to $r=100 \mathrm{~T} \Omega$ with input capacitances less than $20 \mathrm{pF}$. For a $100 \mathrm{~T} \Omega$ input resistance, the voltage measurement can be

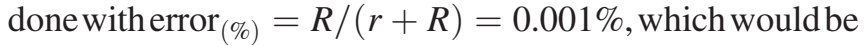
dominated by the instrument's specified accuracy.

Therefore, contact devices such as an electrometer need to provide ultrahigh-input impedance $Z_{i}$ in order to minimize the current flow between the experiment and the device. $Z_{i}$ is determined by the loading impedance of the voltage-sensing electrode of the devices as well as the loading impedances associated with shields and cables connecting the voltage sensor to the electronic circuits. The ultimate goal is to achieve an infinite input resistance and a zero input capacitance.

In the model 800 INFINITRON nonloading voltmeter developed recently by TREK Inc., this is implemented by the use of a guarding (shield) technique, where the electric potential of the shielding system is feedback driven to that of the voltage sensor [7]. In addition, the electrometer has beneficial features assuring that the potential of the sensor and the potential of the OUI are equal (bootstrap 
technology), thus preventing transfer of electric charges. Moreover, the voltmeter is capable of nulling the input bias current to prevent the sensor capacitance charging before making contact, as well as excluding the bias current flow after the contact has been made. An important part of the bootstrapping system is the location of the front end amplifier in the probe body. This eliminates the prospect of ac and dc pickup in the probe cable. Extremely low input capacitance and high input resistance precision circuits are adversely affected by the input offset voltage and the input bias current associated with the input amplifier. These factors have been compensated by implementing corresponding correction circuits.

High-performance electrometers are also available from Keithley. In this work, the model 617 programmable electrometer was used. In Keithley devices, the reduced leakage currents and low-input capacitance are achieved by guarding. The built-in voltage source simplifies determination of the relationship between the resistivity and voltage applied. It is also well suited for the insulation resistance measurement. Table II summarizes the instrument performance for both the model 800 INFINITRON and Keithley model 617 used in this work.

In order to conduct noninvasive tests, a constant voltage method was employed. In this constant voltage method, a known voltage is applied in parallel with the resistor chain and the electrometer is used to measure the voltage distribution across the chain. A second electrometer ammeter or a picoammeter is placed in series with the voltage source to measure the resulting current. The basic configuration of the method is shown in Fig. 5. Since the voltage drop across the electrometer ammeter $\mathrm{A}$ is negligible, essentially all the voltage appears across the resistor chain. The resulting current is measured by the ammeter and the total resistance of assembly is calculated using Ohm's law, $R_{\Sigma}=V / I$.

Measuring high resistance is challenging but can be achieved using an electrometer and proper technique. To make these measurements, an electrometer $\mathrm{E}$ and a constant voltage source are required. Some electrometers such as model 617 have a constant voltage source built into the instrument. For accurate measurements, the highimpedance terminal of the electrometer is always connected to the high impedance of the circuit being measured. If not, erroneous measurements may result. The main application of this setup is noninvasive simultaneous, two-terminal testing of an electrostatic accelerator high-voltage grading system, measuring insulation resistance or determining the volume and surface resistivity of insulation materials used in column posts and acceleration tubes. In addition, it allows verification the continuity of the resistive divider assembly as a complete circuit, determining if an electrical path is established between equipotential rings, resistors, tube electrodes, and post-to-tube conductors.

High resistance measurements on two terminal devices should be done in controlled conditions and are often dependent on time. The capacitor associated with the insulators must be allowed to charge fully by the applied voltage before the measurement is made. Otherwise, the erroneous current of much higher value will be measured. A typical time of capacitor charging is seven time constants, which would allow for $0.1 \%$ of the final value.

Knowing the sources of measurement error and taking steps to eliminate them is essential to making meaningful high-resistance measurements. Electrostatic interference occurs when charged and uncharged objects are brought close together. Since charge in a high-resistance system does not decay quickly, an erroneous measurement may result due to ac or dc electrostatic fields. The dc fields can be detected when body movements near the accelerator structure cause fluctuations on the voltage reading of electrometer and this is usually the case with an accelerator structure. Another quick check for interference would be to place a piece of a charged plastic near the object under investigation and observe a change in the electrometer readout. Equally troublesome are ac fields, which are most often caused by mains power lines and rf fields. Part of this signal is rectified causing the dc offset in the measured signal. This can be verified by observing the analogue output of electrometer with an oscilloscope. To reduce the effects of the fields, an electrostatic shield can be built to enclose the circuits being measured. The shield should be made of conductive material and connected to the low impedance input of the electrometer as indicated by $\mathrm{LO}$ in Fig. 5. The cabling in the circuit must also be low noise shielded coax and triaxial cabling available from both TREK and Keithley. Since the shielding of the bulky accelerator resistor grading system is not practical, a number of strategies were employed to address dc and ac noise. First, all unnecessary power equipment was turned off and power cables and extension leads removed from the accelerator maintenance platform in order to reduce the effect of ac fields. Second, only two operators were allowed to conduct the measurements on the platform and their movements near the experiment were limited according to the measurement protocol.

TABLE II. High-performance electrometer specifications.

\begin{tabular}{lcccccc}
\hline \hline Model & Range, $\mathrm{V}$ & Bandwidth $\left(10 \mathrm{~V}_{p-p}\right)$ & Accuracy & Drift, $\mathrm{mV} / \mathrm{hr}$ & Input capacitance & Input resistance \\
\hline 800 & \pm 100 & $10 \mathrm{kHz}$ & $\pm 0.1 \%$ & $<500$ & $<10^{-15} \mathrm{~F}$ & $>10^{16} \Omega$ \\
617 & 200 & - & $0.4 \%$ & - & $<2 \times 10^{-12} \mathrm{~F}$ & $>2 \times 10^{14} \Omega$ \\
\hline \hline
\end{tabular}




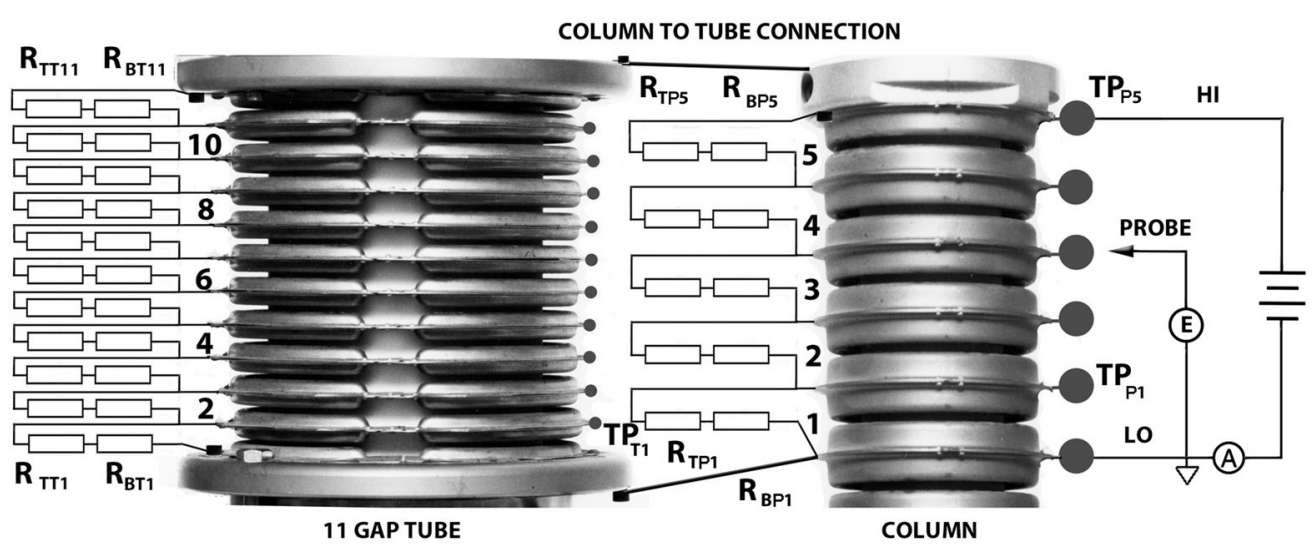

- TEST POINT ON ACCELERATION TUBE

TEST POINT ON EQUIPOTENTIAL RING

FIG. 5. The constant voltage method for noninvasive diagnostics of the high-voltage grading system of an electrostatic accelerator. The 11 gap acceleration tube and the 5 gaps of the top section of the column post are shown. There is a pair of $40 \mathrm{kV}$, $112 \mathrm{~mm}-\mathrm{long}$ Welwyn resistors for each post gap shown as top and bottom resistor $R_{\mathrm{TPi}}$ and $R_{\mathrm{BPi}}$ correspondingly where $i$ is the gap number. The tubes employ pairs of $20 \mathrm{kV}, 61 \mathrm{~mm}$-long $300 \mathrm{M} \Omega$ resistors shown as top and bottom resistors $R_{\mathrm{TTi}}$ and $R_{\mathrm{BTi}}$ correspondingly. The digits shown next to the tube and post are the insulating gap numbers. $\mathrm{E}$ is the electrometer and $\mathrm{A}$ is the second electrometer or picoammeter.

There are a couple of minor effects, which may be a source of measurement errors. A triboelectric current is generated when frictional motion exists between a conductor and its surrounding insulator, causing an error current to flow. For example, this effect occurs when insulators and conductors rub together such as in a coaxial cable. The triboelectric effect was reduced by minimizing mechanical vibrations during measurements and by properly securing low noise cabling between the electrometer and its active sensor. Noise currents can also be generated by a contaminated insulator between two conductors of dissimilar metals due to electrochemical effects. To avoid errors caused by this effect, all insulators of the measuring equipment were maintained in clean conditions and the humidity kept low.

Care must be taken to avoid touching the tip of the electrometer sensor so it does not become contaminated with body oils. For instance, the sensor for model 800 INFINITRON is a 0.05 -inch gold plated rounded tip electrode. It contains a retractable spring mechanism to help absorb initial contact pressure. According to the service manual, isopropyl (anhydrous, 99\%) is used to clean the probe tip contact and its body if it becomes contaminated. Noninvasive tests would not be possible without development of the two adaptor devices to the sensor in order to sample post and tube test points shown in Fig. 5. The first adaptor " $\mathrm{R}$ " allows reliable and reproducible positioning of the sensor on the equipotential ring. The second adaptor " $\mathrm{T}$ " was inserted between neighboring equipotential rings in order to make electrical contact between the sensor and electrode on the acceleration tube. A V-grove is machined at the tip of the adaptor " $T$ " providing a self-aligned guide for quick mechanical engagement of the probe with the flat surface of the tube electrode. High-quality nylon 6 insulator is used when manufacturing the adaptors. Both adaptors " $\mathrm{R}$ " and " $T$ " are shown in Video 1 during measurements on the one unit of the 14UD.

The measured voltage distribution across an 11 gap tube and the corresponding 5 column post gaps is shown in Table III. Test points on the tube $\mathrm{TP}_{T}$ and on the post $\mathrm{TP}_{P}$ are marked the same way as shown in Fig. 5. Similar tables were produced for the 8 gap tube and the 6 gap post configurations.

The measurement set up shown in Video 1 illustrates the configuration for an 11 gap tube. A voltage of $40 \mathrm{~V}$ is applied to 5 gaps on the post by connecting cable leads to

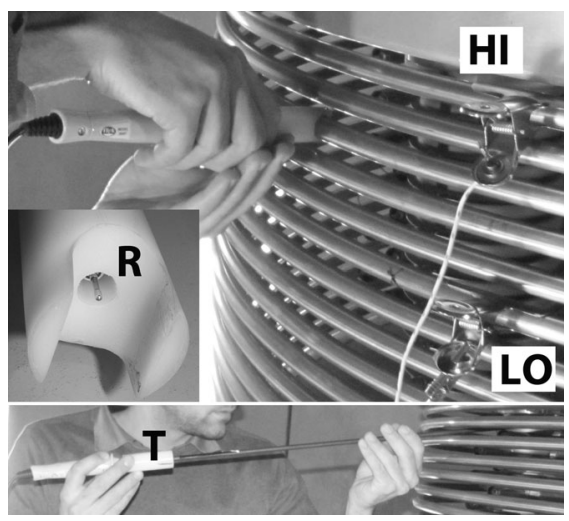

VIDEO 1. Adaptor devices developed for the INFINITRON 800 sensor for noninvasive resistance measurements. Adaptor " $\mathrm{R}$ " is designed for reproducible positioning of the sensor on an equipotential ring. Inset shows the front end of the adaptor with sensor tip protruding. Adaptor " $\mathrm{T}$ " is used as an insertion device for quick access to the electrodes on an acceleration tube. 
TABLE III. Measured voltage distribution across an 11 gap acceleration tube and the corresponding 5 gaps on the column post.

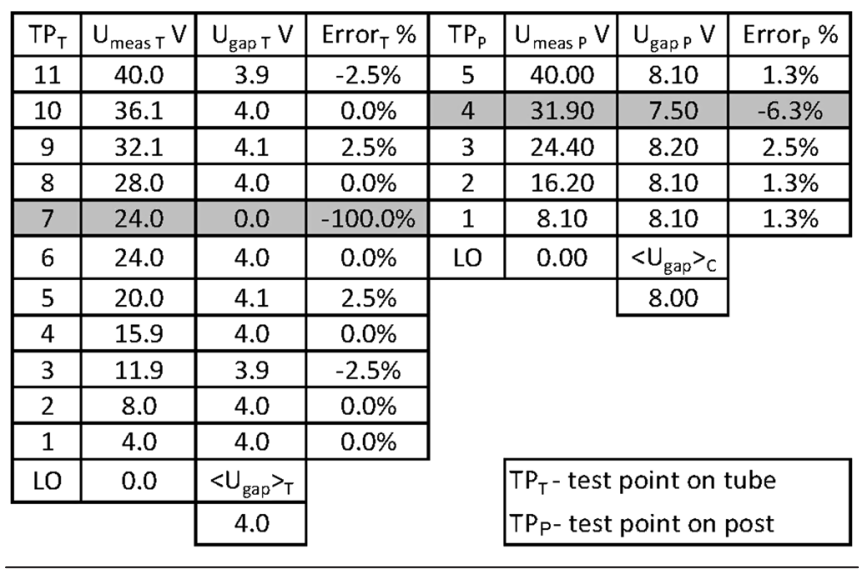

equipotential rings marked as $\mathrm{LO}$ and $\mathrm{HI}$. The same voltage is applied to the corresponding top and bottom gaps of the 11 gap tube via post-to-tube wire conductors that run from one of the four outer posts in toward the acceleration tube. The voltages at each test point $\left(U_{\text {meas } T}\right.$ or $U_{\text {meas } P}$ on the tube and post, respectively) were read from the electrometer display and recorded. The voltage drop per tube, $U_{\mathrm{gap} T}$, or post gap, $U_{\mathrm{gap} P}$, was then calculated leading to a mean value of voltage drop per gap $\left\langle U_{\text {gap } T}\right\rangle$ and $\left\langle U_{\text {gap } P}\right\rangle$. Any gap voltage deviation $\Delta U_{\text {gap }} / U_{\text {gap }}> \pm 2.5 \%$ were excluded from calculation of $\left\langle U_{\text {gap }}\right\rangle$. As discussed in the Introduction, good resistor performance is indicated by a relative resistance change $\Delta R / R< \pm 2.5 \%$. The error value was calculated by using the formula $\%$ error $=100\left(U_{\text {gap }} /\left\langle U_{\text {gap }}\right\rangle-1\right)$. Typically it took less than 4 min to collect data related to one tube and corresponding section of the post. It required less than $14 \mathrm{~min}$ to measure a complete 14UD unit and about $6.5 \mathrm{hr}$ to compile the voltage distribution of the entire accelerator

Consider a chain of $N$ identical resistors of value $R$ in series with applied voltage $U_{\text {meas }}$. If the value of the single resistor is changed by $\Delta R$, the voltage drop across this resistor is $U_{R+\Delta R}=U_{\text {meas }}(\Delta R+R) /(\Delta R+N R)$. The voltage drop across an unchanged resistor is $U_{R}=$ $U_{\text {meas }} R /(\Delta R+N R)$. Therefore the voltage difference is given by $\Delta U=U_{R}-U_{R+\Delta R}$ and the relative resistance change is then

$$
\Delta R / R=\Delta U \times N /\left(U_{\text {meas }}-\Delta U\right) .
$$

The resolution of this method is limited by the electrometer accuracy of the voltage measurement, $\Delta U / U=0.1 \%$. For an 11 gap tube structure with $N=11$ and $U_{\text {meas }}=$ $40 \mathrm{~V}$, the calculated $\Delta R / R_{11 G T}=2.8 \%$. For the corresponding 5 gap post structure with $N=5$ and the same test voltage, the calculated $\Delta R / R_{5 G P}=1.25 \%$. The $\Delta R / R$ resolution achieved with a high-impedance electrometer is much better than is possible using a $30 \mathrm{kV}$ group test technique. In addition, it can be improved even further by operating at $U_{\text {meas }}=100 \mathrm{~V}$ after implementation of appropriate safe working procedures.

Voltage distribution tables with calculated errors provide additional information to localize faults. Evaluation of the data presented in Table III provides a feel of what is going on in the high-impedance circuit under examination. The components exhibiting error below $\pm 2.5 \%$ were passed as free of faults. Components with measured error above $\pm 2.5 \%$ were considered faulty and required further examination. In the example results presented in Table III, two faults are highlighted. It suggests that there is a short circuit in the seventh tube gap indicated by zero voltage across this gap. It can be caused by catastrophic failure of the resistor, shorting of the tube ceramic gap or by conductive debris across tube or resistor sparking gap. There is also low resistance across the forth post gap. The cause may be a partially short resistor, current leakage through a post insulator, or by the presence of moisture. The challenge is to decide which measurement to make as a next step. The process of fault finding within a high-voltage grading system has been developed over the last couple of years in our laboratory. It is based on a few fundamental examples and was developed into simple concepts providing practical tips and hints for successful localization of faults as shown in Fig. 7.

In Fig. 7 the vertical axis is the normalized voltage distribution $U / U_{\max }$ across 5 gaps under test. The first graph (1) shows the test outcome when the system is considered to be fault free. The calculated error value is within the range $\pm 2.5 \%$. No further action is required. Graph (2) is the case when an open circuit has been found in gap 3, for which the error value becomes infinity. The possible corresponding failure modes listed in Table I are A, B, D, and E. A short circuit across gap 3 is shown in Fig. 7 (3) with error $=-100 \%$. This case is relevant to failure mode G. Graphs (4) and (5) with error above $\pm 2.5 \%$ point to a significant change of resistance in gap 3. This could result from a change of high-voltage resistance value (failure mode $\mathrm{C}$ and $\mathrm{F}$ ), wrong resistor value due to fault during assembly (failure mode $\mathrm{O}$ ), insulator failure (failure mode $\mathrm{H}$ ), and insulator leakage (failure modes I and J). Finally, graph (6) is an open circuit in gap 1. It is similar to (2) in that the possible failure modes could be A, B, D, and E. In addition, an open circuit associated with the first and last gap of the unit section under test may also indicate failure of external conductors and connectors as in failure modes $\mathrm{K}, \mathrm{L}, \mathrm{M}$, and $\mathrm{N}$. Table IV summarizes the fault conditions.

The analysis of a particular pattern collected during the primary noninvasive test determines what measurement to make as a next step. Units with faulty components will be opened (equipotential rings removed) to gain access for 

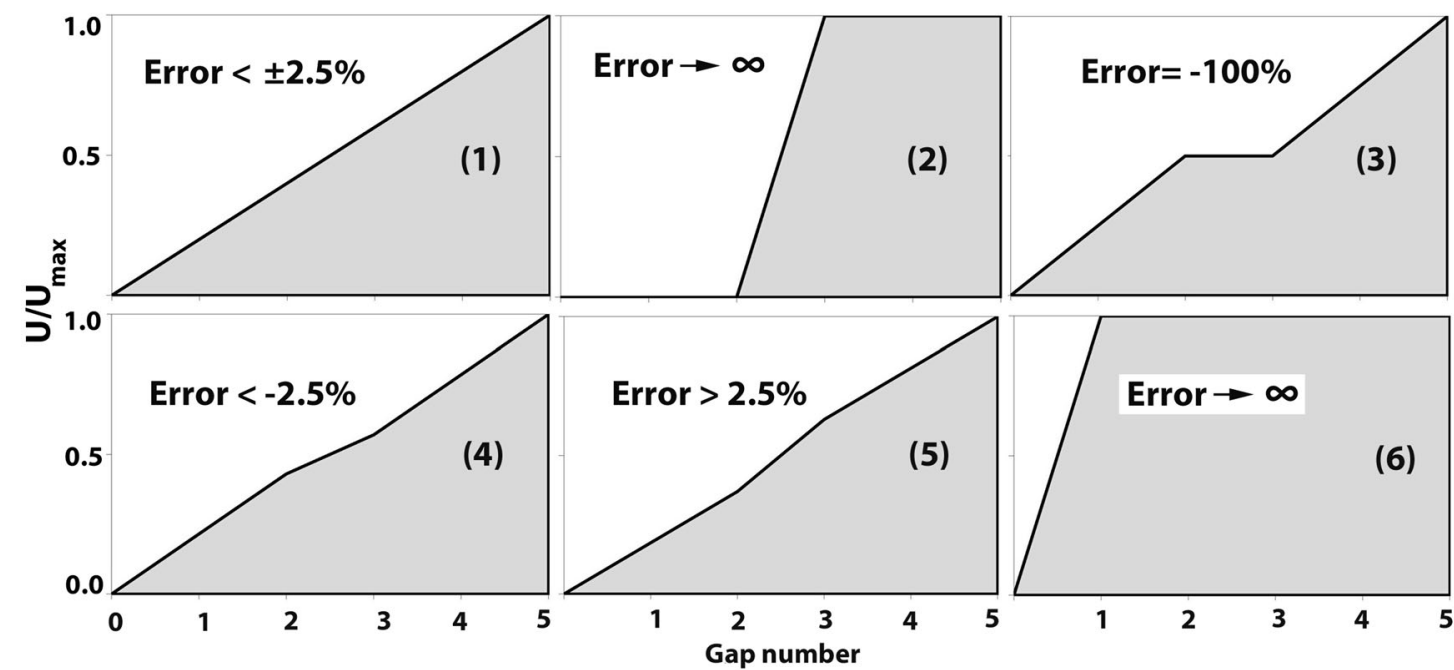

FIG. 7. Troubleshooting chart for express identification of faults in the high-voltage grading system of an electrostatic accelerator based on a noninvasive test with an electrometer. The vertical axis for all graphs is the normalized voltage distribution across 5 gaps corresponding to different fault conditions such as (1) fault-free system; (2) open circuit in gap 3; (3) short circuit in gap 3; (4) lower resistance in gap 3; (5) higher resistance in gap 3, and (6) open circuit in gap 1. A 5 gap structure is selected for simplicity and illustration purposes. The same analysis is applied to any structure with arbitrary number of gaps under test.

further "invasive" investigation. At this stage, all diagnostics techniques listed in Table I could be employed to identify any resistor and insulator failure mode.

Identification and location of a microbreak is only possible using an electrometer. A resistor suspected to have a microbreak is removed from the accelerator and disassembled. The resistor is fixed into a positioning stage and $40 \mathrm{~V}$ is applied across the resistor. A voltage distribution along the resistor is sampled by scanning along the conductive path with an electrometer probe. Figure 8 shows the probe tip in contact with the conductive thin film layer of the resistor.

Measurement begins from the resistor end connected to LO terminal of the power supply. The microbreak manifests as a sudden jump of the measured voltage from zero to the supply maximum of $40 \mathrm{~V}$. The exact location of the microbreak is revealed by visual inspection under a microscope in order to reveal its microstructure and cause.

This same setup was used for initial acceptance and qualification tests of new Welwyn resistors recently

TABLE IV. Troubleshooting chart for identification of faults in high-voltage grading system of electrostatic accelerators.

(6)

\begin{tabular}{lccccc}
$(1)$ & $(2)$ & $(3)$ & $(4)$ & $(5)$ & Open circuit \\
Fault & $\begin{array}{c}\text { Open } \\
\text { free }\end{array}$ & $\begin{array}{c}\text { Short } \\
\text { circuit } \\
\text { circuit }\end{array}$ & $\begin{array}{c}\text { Lower } \\
\text { resistance }\end{array}$ & $\begin{array}{c}\text { Higher } \\
\text { resistance }\end{array}$ & $\begin{array}{c}\text { in the first } \\
\text { or last gap }\end{array}$ \\
\hline - & A, B, & G & C, F, & C, O & $\begin{array}{c}\text { as (2) plus } \\
\text { D, E }\end{array}$ \\
& & I, J, O & & K, L, M, N \\
\hline \hline
\end{tabular}

purchased for spares. These resistors passed the resistivity test. However, visual inspection revealed nonuniform distribution of the width of the helix conductive layer as shown in the inset of Fig. 8. This pattern could result in a nonuniform voltage distribution along the resistive layer, creating localized areas of excessive voltage gradient, potentially leading to premature failure. An electrometer could be used by sampling voltage distribution across the resistor in order to quantify nonuniformity.

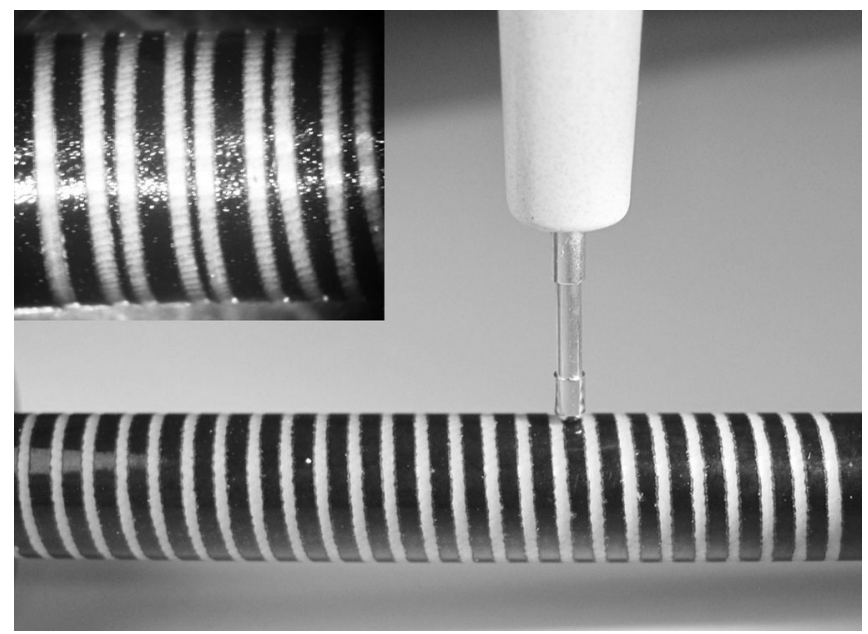

FIG. 8. Experimental setup for measuring the voltage distribution across a gigaohm resistor to locate a "microbreak." A $982 \mathrm{M} \Omega$ Welwyn resistor and an INFINITRON Model 800P probe are shown. The inset shows the Welwyn resistor with a nonuniform distribution of the width of the helix conductive layer (dark). 


\section{RESULTS}

The experimental results described in this paper were collected during five scheduled accelerator maintenance tank openings (TOs) starting from TO \#119 when the electrometer was first commissioned in February 2013, up to TO \#123 in July 2014. Tank Opening Reports (TOR) are available from [8].

Figures 9 and 10 illustrate the electrometer results of entry and exit tests of the accelerator resistive grading systems associated with tubes and column posts. The entry test establishes the initial state of the accelerator, which depends on previous operational conditions such as maximum working voltage, spark event frequency, beam loading, and conditioning status. The aim of the exit test is to confirm that the resistive grading system is well balanced within $\pm 2.5 \%$ of the acceptance margin after appropriate maintenance has been performed.

The top bar chart in Fig. 9 shows the compilation of entry test distributions of absolute value of maximum relative resistance deviation $\left|\left(\Delta R_{P} / R\right)_{\max }\right|$ in the post structure linked to the acceleration tube by means of post-to-tube connection wires.

The horizontal axis is the number of post sections linked to the accelerating tube number from the low-energy to the high-energy end of the machine. The entry test includes measurements carried out during TO \#119 to TO \#123. The bottom bar chart in Fig. 9 represents the exit test distribution of $\left|\left(\Delta R_{P} / R\right)_{\max }\right|$ measured at the end of the TO \#123.

The top bar chart in Fig. 10 displays the compilation of entry distributions of absolute value of maximum relative resistance deviation $\left|\left(\Delta R_{T} / R\right)_{\max }\right|$ in the acceleration tubes. The horizontal axis is the number of accelerating tubes starting from the low-energy to the high-energy end. The bottom bar chart in Fig. 10 denotes the exit test distribution of $\left|\left(\Delta R_{T} / R\right)_{\max }\right|$ assessed on completion of maintenance work at the end of the TO \#123.

Components with measured $\Delta R_{T} / R> \pm 2.5 \%$ were considered faulty and put through further detailed examination using the methodology discussed in the previous section. Classification of the observed faults is shown in the Pareto chart shown in Fig. 11.

A Pareto chart is one of the basic tools of quality control. It highlights the concept of the 80-20 rule, which reveals the failure modes that should be addressed to take care of $80 \%$ of the faults. In total, 34 faults were identified and most of them would not be possible to discover without the electrometer-based diagnostic technique. Category $H_{p}$ failures-postmechanical failure-were
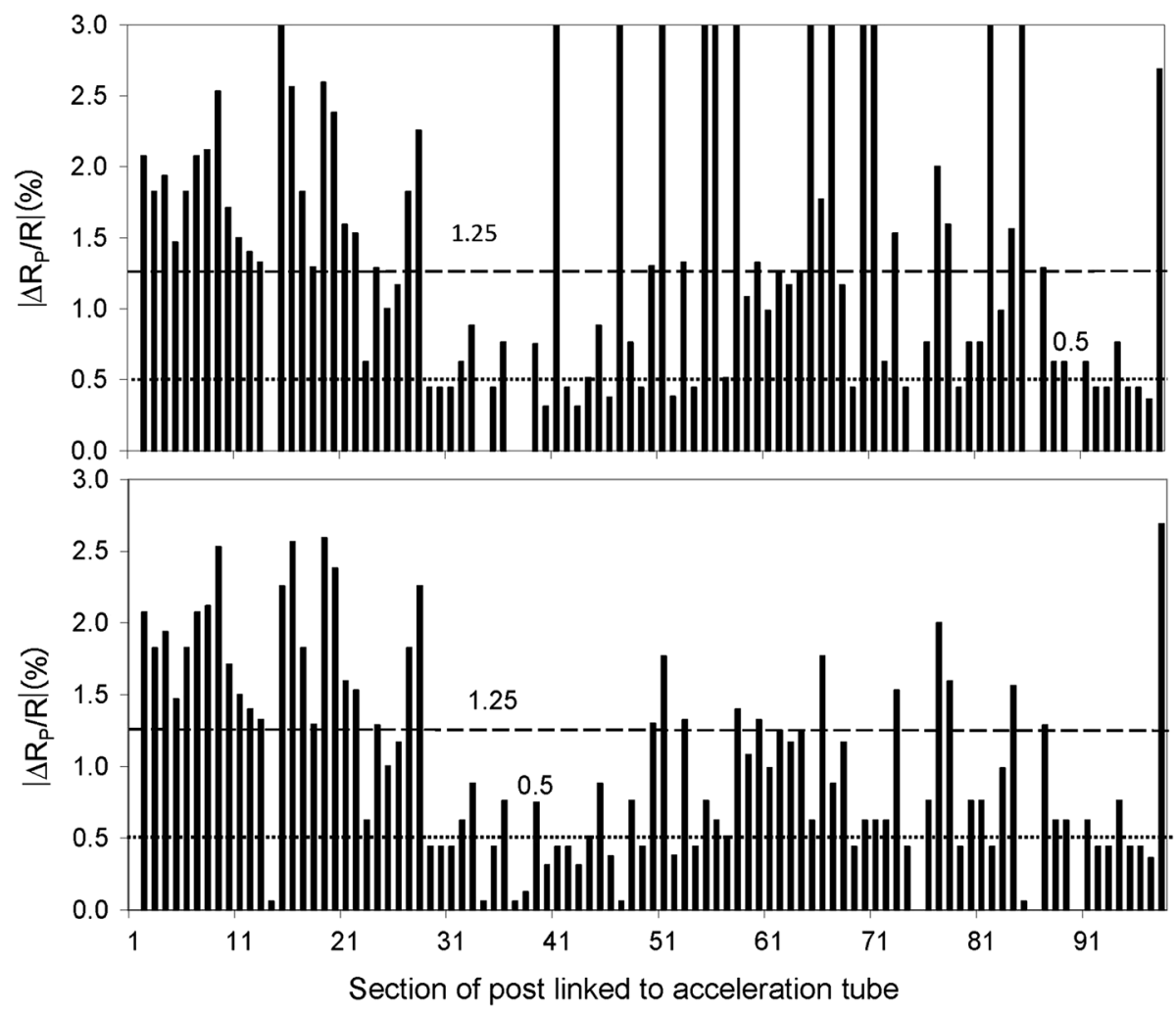

FIG. 9. The distribution of absolute value of maximum relative resistance deviation $\left|\left(\Delta R_{P} / R\right)_{\max }\right|$ in the post structure linked to the acceleration tube. The horizontal axis is the number of the post section linked to the accelerating tube starting from low energy to high energy. The top chart is the compilation of entry test distributions accumulated during five tank openings starting from TO \#119 to TO $\# 123$. The bottom chart is the exit distribution collected in the end of TO \#123. Dashed line represents $1.25 \%$ resolution of electrometer technique if measurements are carried out at $40 \mathrm{~V}$. Dotted line corresponds to $0.5 \%$ resolution if operating at maximum voltage of $100 \mathrm{~V}$. 

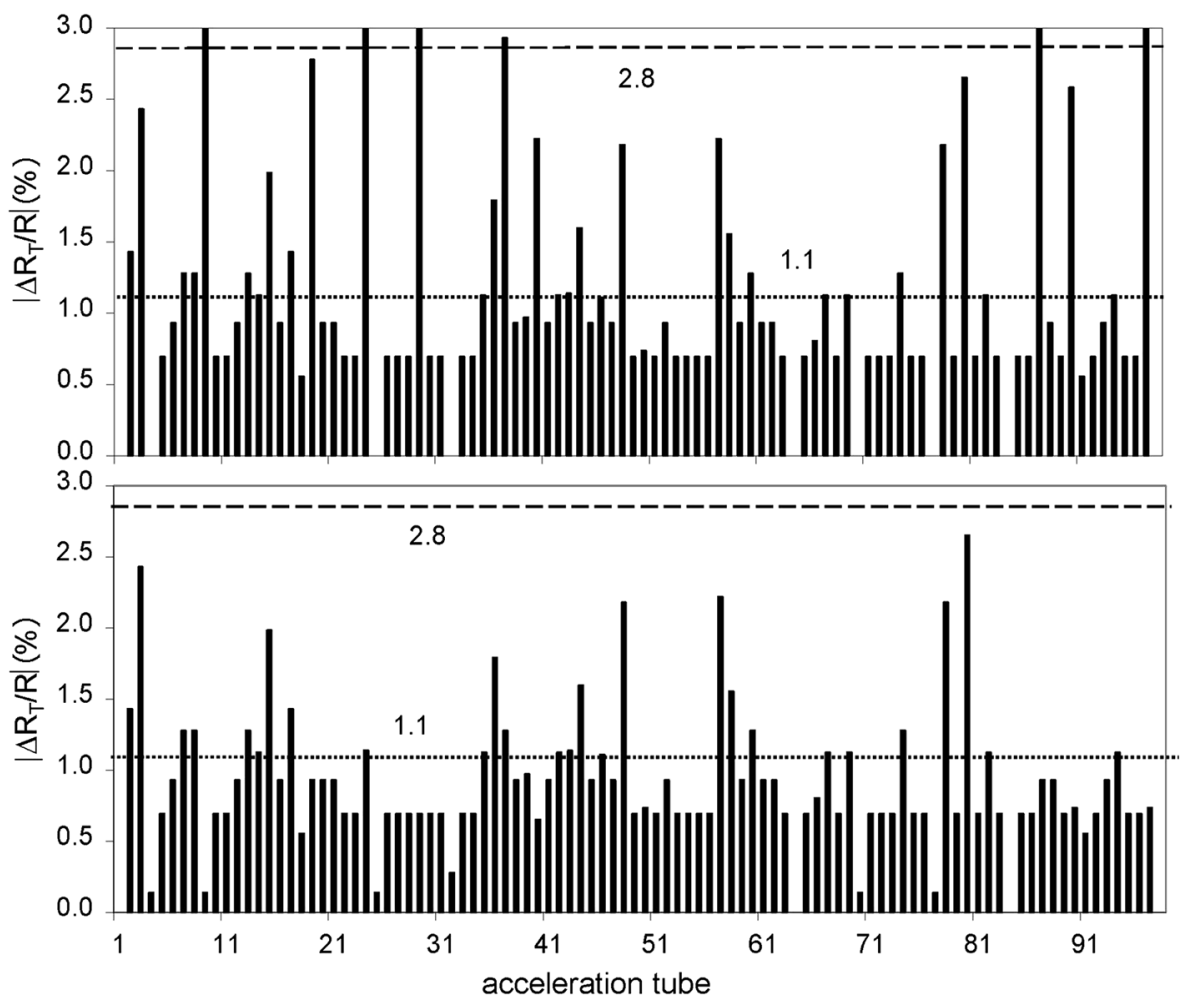

FIG. 10. The distribution of absolute value of maximum relative resistance deviation $\left|\left(\Delta R_{T} / R\right)_{\max }\right|$ in the accelerating tubes. The horizontal axis is the number of the accelerating tube starting from low energy to high energy. The top chart is the compilation of entry test cumulative distribution accrued during five tank openings starting from TO \#119 to TO \#123. The bottom chart is the exit distribution collected in the end of TO \#123. Dashed line displays 2.8\% maximum resolution of electrometer-based technique providing measurements are carried out at $40 \mathrm{~V}$. Dotted line indicates limit of $1.1 \%$ resolution if operating at maximum voltage of $100 \mathrm{~V}$.

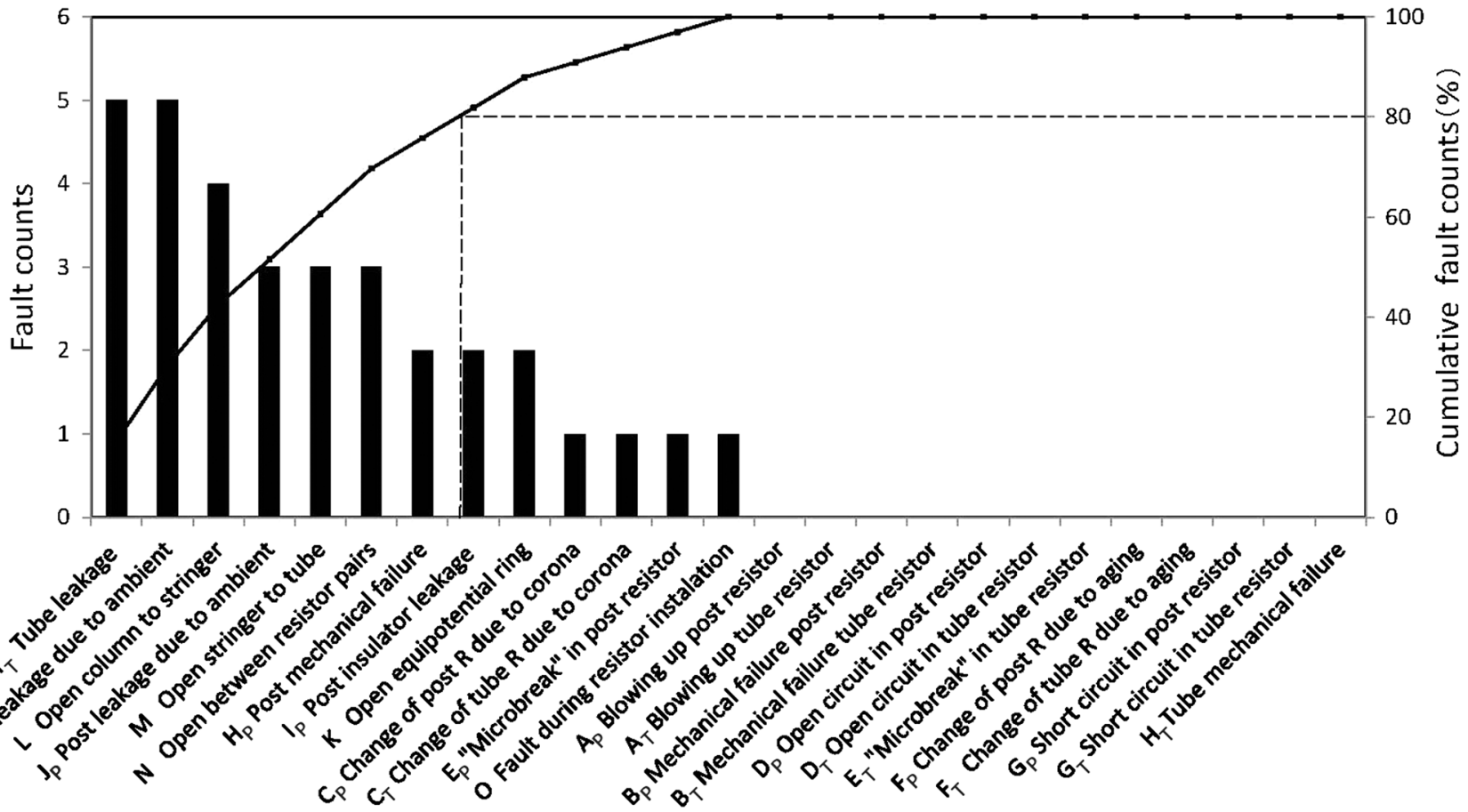

FIG. 11. Pareto chart of the number of 14UD electrostatic structure fault occurrence as discovered during the maintenance five tank openings spanning from TO \#119 to TO \#123. The dashed line starting at 80\% of the right $y$ axis highlights $80-20$ rule. Subscript letters "T" and "P" are used to refer to accelerating tube or post correspondingly. 
discovered accidentally. Unfortunately none of the conventional and new techniques as listed in Table I had any response to this important malfunction of the main insulating structural component of the accelerator.

\section{DISCUSSION}

Overall, it is immediately apparent from the entry test results presented in Figs. 9 and 10 that the maximum relative resistance deviation $\left|\left(\Delta R_{P} / R\right)_{\max }\right|$ in the post structures is considerably higher compared to $\left|\left(\Delta R_{T} / R\right)_{\max }\right|$ observed in the accelerating tubes. At first glance, one can see that posts with an unacceptably high value of $\left|\left(\Delta R_{P} / R\right)_{\max }\right|>2.5 \%$ are more likely to be located in the high-energy end of the 14UD accelerator, corresponding to section numbers from 50 to 98 . However, the tube resistance deviations with $\left|\left(\Delta R_{T} / R\right)_{\max }\right|>2.5 \%$ are more common in the low-energy end, with the section numbers from 1 to 49 .

According to the Pareto chart shown in Fig. 11, the most common failure modes are tube insulator leakage $\left(I_{T}\right)$ and tube insulator current developed after being exposed to an ambient environment $\left(J_{T}\right)$, at around $15 \%$ each. Faults falling under category $\left(I_{T}\right)$ might be caused by progressive creation of a conductive path along a surface due to the spark gap firing or surface flashover. Nevertheless, a number of tubes had surface marks and discoloration on the ceramic insulator between titanium electrodes as shown in Fig. 12 and none showed any current leakage when $6 \mathrm{kV}$ was applied across the gap.

A consistent pattern is also seen with tube or post ceramic in that any gap that exhibits current leakage has at least a hairline crack in the insulator as per category $\left(J_{T}\right)$. The leakage develops after a component is exposed to the ambient environment and the crack preferentially absorbs moisture due to a capillary effect. Components with cracks in ceramic were baked out overnight at $80^{\circ} \mathrm{C}$ and showed no leakage. It is possible that when the same component is exposed to a dry $\mathrm{SF}_{6}$ environment, as is the case during normal accelerator operation, the moisture is also driven out and the leakage current disappears.

The next most commonly observed fault category is L, lack of continuity or poor electrical connection between
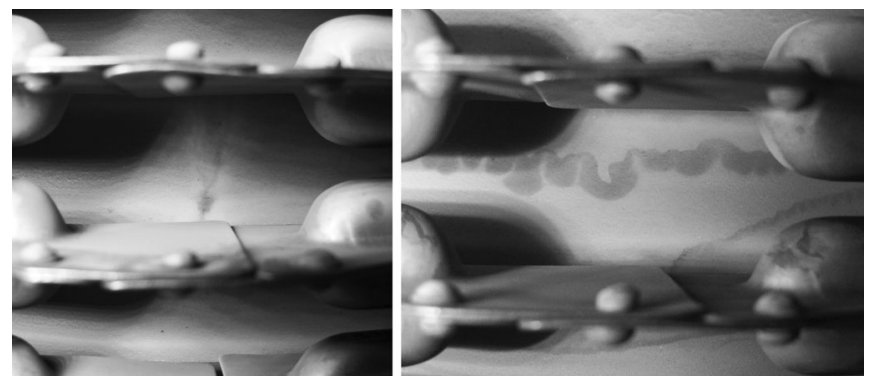

FIG. 12. Two examples of arc track marks in accelerating tubes which did not exhibit any surface leakage after applying a $6 \mathrm{kV}$ test voltage across the gap between titanium electrodes. post electrode and post-to-tube conductor (stringer) at approximately $12 \%$. Failures of the rivet style mounting are the common cause. In the worst case, rivet holes are burnt through, resulting in no rivet mounting option left without complete replacement of a post. A new stringer mount is designed based on resistor clamps as shown in Fig. 13.

The next important fault is the leakage current in the post insulator developed after the component is exposed to the ambient environment $\left(J_{P}\right)$ with weight factor at around $12 \%$. This phenomenon occurs because of similar hairline cracking in the tube ceramic as discussed for $\left(J_{T}\right)$. However, with posts the type of damage was not limited to hairline cracks but also included surface flaking and crisscrossed cracks.

Lack of continuity between stringer and tube electrode, fault type $\mathrm{M}$, is another relatively common fault at about $12 \%$. Loose stringer screws were found in a few units and a better option for fastening was developed. Smoothed hex socket screws were introduced instead of the button head used previously as shown in Fig. 13. The larger key size allows more torque to be applied for better tightening and better electrical contact between the stringer and tube. These new arrangements will be monitored and assessed as a long-term solution.

Next is the open circuit or poor continuity between pairs of resistors, N, with cumulative fault proportion of $12 \%$. Pairs of resistors are connected together with a wire attached to the nut and banana plug socket machined as a part of the nut. An image of a burnt-out resistor plug is shown in Fig. 14(a). The design has proved to be very successful based on operational performance over the last two decades. Nevertheless, spark damaged wire leads are still found and easily replaced.

The most serious problem is the mechanical damage of post ceramics $\left(H_{P}\right)$, since they form crucial structural components of the accelerator. A complete separation of the bond between the ceramic and titanium electrode was found on two occasions as shown in Fig. 14(b). Posts were

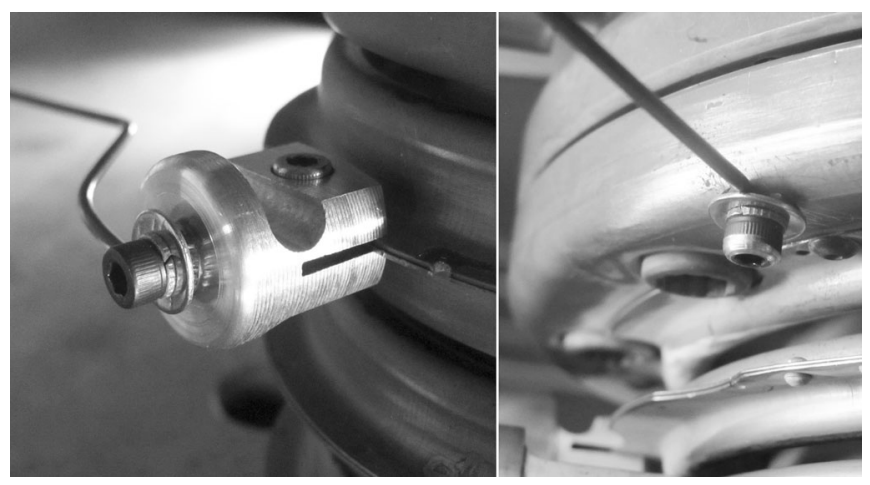

FIG. 13. New stringer: post mount using resistor clamp (left) and high-torque rounded hex head screw used to attach the stringer to tube (right). 


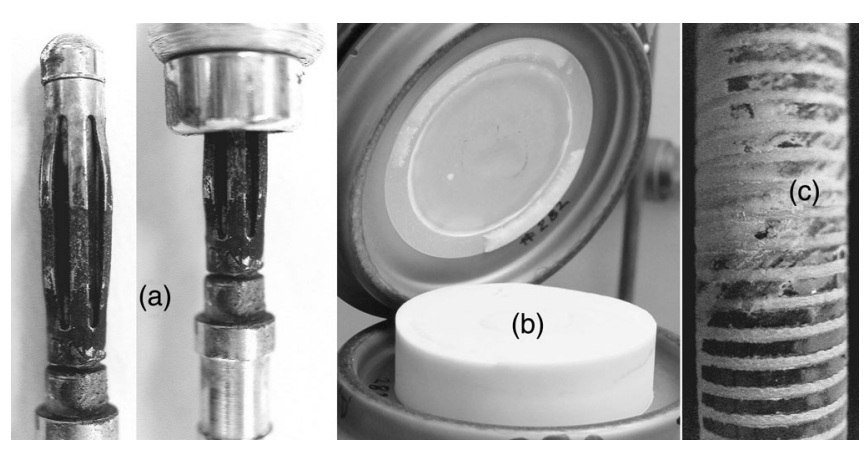

FIG. 14. (a) Spark-eroded resistor plug connecting flexible lead to resistor circuit $(\mathrm{N})$ and the same plug inserted partially into resistor receptacle. (b) Complete separation of the bond between the post ceramic and titanium electrode $\left(H_{P}\right)$. (c) Erosion of conductive layer of post resistor due to exposure to corona discharge $\left(C_{P}\right)$.

separated in two pieces once released from their compressive load of the structure. Ceramic failures could be a dangerous and high-cost occurrence. This calls for the development of a suitable technique for in situ structural diagnostics of post ceramics.

The remaining faults are affiliated with the following categories: (i) open circuit in electrical path between equipotential ring and post electrode $(\mathrm{K})$; this has been confirmed with a continuity meter and fixed by mechanical adjustment of the ring shape; (ii) variation of tube or post resistor value due to exposure to corona discharge caused by erosion of conductive layers $\left(C_{P}\right)$ and $\left(C_{T}\right)$ as presented in Fig. 14(c); (iii) microbreak $\left(E_{P}\right)$ which has been already discussed in the Introduction; and (iv) an error during resistors installation $(\mathrm{O})$.

It was discovered that the first three and last three pairs of post resistors in unit 20 had been installed at the wrong ends of the post. Thus the post resistors associated with the 11 gap tube and 8 gap tube were incorrect; see Fig. 2. This occurred by mistake when the resistors were removed and replaced during the installation of a new column post in 2005.

The total proportion of faults for tubes is $32 \%$ and $27 \%$ for posts, with insulator leakage current being the most common fault. This higher proportion of faults on acceleration tubes was unexpected, since tubes are usually better protected when compared to posts. On the other hand, posts and their resistors are more accessible and historically have been replaced more often as a part of a post refurbishing program. This failure pattern may be specific for the ANU 14UD electrostatic accelerator, reflecting its usage, maintenance procedures, and age.

Finally, the bottom bar charts in Figs. 9 and 10 denote the exit test distribution of the absolute value of maximum relative resistance deviation of accelerating tube $\left(\Delta R_{T} / R\right)_{\max }$ and post $\left(\Delta R_{P} / R\right)_{\max }$ assessed on completion of maintenance work at the end of the TO \#123. Overall, it can be seen that as a result of in situ troubleshooting and fault elimination, the maximum values of $(\Delta R / R)_{\max }$ are kept below $2.5 \%$. The acceptance margin could be narrowed even further by a factor of 2.5 by increasing the setup test voltage up to $100 \mathrm{~V}$. The heavy ion physics program at ANU requires the highest available energies from the heavier ions. Since the tandem is also injecting a booster, additional requirements call for high stability of the energy and phase of pulsed beams. The ANU's 14UD typically runs for $6000 \mathrm{hr}$ per year, achieving beam on target for about $80 \%$ of that time. There are between one and two tank openings per year, with the frequency determined by stripper foil life or the routine maintenance of mechanical devices or electrical structures. Mechanical failures of chains or bearings and electrical faults are the main causes for unscheduled maintenance, but resistor and insulator failures are less common now due to routine use of a noninvasive ultrahigh-impedance technique.

\section{ACKNOWLEDGMENTS}

This work has been supported by the Australian Federal Government Superscience/EIF funding under the NCRIS mechanism. The authors have benefited from discussions with many colleagues. However, particular thanks are due to K. Fifield and D. C. Weisser. We wish to express our appreciation to RSPE's Nuclear Physics technical staff members A. Muirhead, J. Heighway, J. Bockwinkel, G. Crook, C. Gudu, and L. Lariosa for their skill and diligence during the 14UD tank openings and tests described in this paper. Crucial design contributions have been made by $\mathrm{T}$. Tunningley for the development of the electrometer's probe adaptors.

[1] T. Joy, Electrostatic accelerator tubes-recent progress and future directions, Nucl. Instrum. Methods Phys. Res., Sect. A 287, 48 (1990).

[2] J. A. Ferry, Recent developments in electrostatic accelerator technology at NEC, Nucl. Instrum. Methods Phys. Res., Sect. A 328, 28 (1993).

[3] D. C. Weisser, Resistor assemblies, their development and performance, Nucl. Instrum. Methods Phys. Res., Sect. A 328, 138 (1993).

[4] J. Noe, in Proceedings of the Symposium of North Eastern Accelerator Personnel, Notre Dame, France, 1986 (World Scientific, Singapore, 1987), pp. 168-185.

[5] C. E. Linder, T. A. Trainor, and W. G. Weitkamp, in Proceedings of the Symposium of North Eastern Accelerator Personnel, Oak Ridge, USA, 1989 (World Scientific, Singapore, 1990), pp. 164-173.

[6] N. R. Lobanov, P. Linardakis, and D. Tsifakis, Development of the fast voltage control method for electrostatic accelerator, Nucl. Instrum. Methods Phys. Res., Sect. A 767, 433 (2014).

[7] M. A. Noras, in Industry Applications Conference, 2005 40th IAS Annual Meeting. Conference Record of the 2005 IEEE, Vol. 3 (IEEE, Hong Kong, 2005), pp. 2194-2197.

[8] http://physics.anu.edu.au/nuclear/tor.php. 